\title{
Polémica sobre César Vallejo
}

\section{SOBRE VALLEJO: RESPUESTA A KEITH A. McDUFFIE}

En un artículo de 26 páginas ("Todos los Ismos el Ismo: Vallejo Rumbo a la Utopía Socialista", Revista Iberoamericana, No. 91, abril-junio 1975, pp. 177202), el Prof. Keith A. McDuffie comenta dos libros en prosa de César Vallejo, Contra el secreto profesional y $E l$ arte y la revolución, recientemente publicados, y se refiere constantemente, con abundante despliegue de citas y notas a veces extensísimas, a un artículo mío ("Vallejo entre la vanguardia y la revolución (Primera lectura de dos libros inéditos)", Hispamérica, No. 6., abril 1974, pp. 3 . 12), dedicado a las mismas obras del poeta peruano. Como el Prof. McDuffie ha consagrado varios trabajos a Vallejo, especialmente a Trilce, que considero muy valiosos, empecé a leer su artículo con interés. Mi interés creció más todavía cuando comprobé que, en su opinión, casi todas las afirmaciones y observaciones hechas en mi artículo, eran erróneas, inexactas o confusas. Por un principio elemental de cortesía entre colegas quiero retribuir esas anotaciones del Prof. McDuffie, mostrándole cuáles son sus errores, sus inexactitudes y sus confusiones.

En primer término, lamento tener que decir que el autor ha comprendido poco de mi artículo que, por otra parte, está escrito en un lenguaje sencillo y sin notas demasiado aparatosas. Mejor dicho: el Prof. McDuffie casi ha leído un texto distinto del. que yo he escrito para oponerle sus propios descubrimientos, recurriendo al cómodo expediente de las citas sueltas y los comentarios in. tercalados, operación interesante pero peligrosa como sistema crítico. El mayor error del-Prof. McDuffie es creer que él ha visto lo que yo he visto y suponer, por lo tanto, que estamos en desacuerdo. Me alegra ahora poder manifestar que tal desacuerdo no existe principalmente sino en la maraña de las citas. Permítaseme ahora algunas aclaraciones.

La primera referencia a mi artículo que deseo aclarar aparece en la nota 8 de la p. 181. Después de citar un texto de Contra el secreto..., el autor escribe en la 
indicada nota:" José Miguel Oviedo no parece entender bien este pasaje cuando lo califica de 'retroceso' a los 'acentos de Trilce' y lo contrapone a otros en que 'razona. dialécticamente' Vallejo. Es nuestro juicio que este pasaje es también dialéctico, como explicamos luego”. La explicación es ésta: “...Vallejo parte de un presupuesto implícito: el del materialismo dialéctico como método filosófico, el que conduce, por medio de una síntesis de contrarios, hacia 'los grandes acordes', frase positiva por completo, que nos hace entender 'panteón' también en forma positiva, quizás en el sentido de los nuevos seglares 'dioses' salvadores de la humanidad, que serían los propios 'grandes acordes'. La palabra clave es 'vana'; nada ni nadie podrá impedir tal proceso. Los 'ayes' nos recuerdan el poema LXXIII de Trilce: 'Ha triunfado otro ay. La verdad está allí'" (p. 182). Como se ve, el Prof. McDuffie, curiosamente, comienza refutándome y luego asume mi tesis: él también, leyendo ese texto, recuerda Trilce, que era lo que me interesaba señalar. Más extraño me parece el método que McDuffie usa para establecer que el pensamiento de este texto es "dialéctico". Creo que no basta afirmar que grandes acordes y panteón son "positivos". La expresión "avanzan así las diferencias de hojas alternas hacia el panteón de los grandes acordes" tiene muchas correspondencias en el lenguaje trílcico: "dos badajos inacordes de tiempo/en una misma campana" (XXXIII); "Rehusad la simetría a buen seguro" (XXXVI); "Cierta guardarropía, sólo ella nos sabrá/a todos en las blancas hojas/de las partidas... Buena guardarropía, ábreme tus blancas hojas... En los bastidores donde nos vestimos,/no hay, no Hay nadie: hojas tan sólo/de par en par" (XLIX); "A veces doyme contra todas las contras,/y por ratos soy el alto más negro de las ápices/en la fatalidad de la Armonía" (LIV); "flotáis nadamente detrás de aquesa membrana que, péndula del zenit al nadir, viene y va de crepúsculo a crepúsculo, vibrante ante la sonora caja de una herida que a vosotros no os duele" (LXX); "temo que ella se vaya, sin haberme probado/en las sequías de increíbles cuerdas vocales,/por las que,/para dar armonía./hay siempre que subir inunca bajar!" (LXXVII), etc. El fragmento en cuestión no está, por lo tanto, inaugurando un nuevo modo poético o de pensamiento; está clausurando uno perfectamente conocido e identificado.

En la parte final de la misma nota 8, el autor agrega: "Afirma [JMO], además, que la obra en su totalidad muestra 'avances y retrocesos que testimonian la complejidad del proceso en el que, como artista y como hombre, Vallejo se ha [sic] envuelto'. A nuestro parecer, el proceso es complejo porque abarca tantos campos de la cultura, no porque hay irresolución por parte del autor" (p. 181). Es muy sorprendente que, en otro lugar de su artículo, al comentar y discutir una afirmación mía sobre las contradicciones ideológicas que estos libros dejan traslucir, el Prof. McDuffie encuentre argumentos para señalar que yo juzgué ese asunto "con la mayor generosidad" (p. 192) y condenar severamente esas contradicciones o "avances y retrocesos" a los que aludía en su nota: "Pero dos consideraciones obran aqui para impedir que a Vallejo se le disculpe por completo la existencia de esas contradicciones. En primer lugar, al parecer existen lado a lado en una sola obra, lo que indica un descuido muy grave 
y hasta un abuso del lector. En segundo lugar, es difícil aceptar lo aparentemente casuístico de tal cambio de actitud respecto a un asunto tan grave como es el de la integridad artística" (p. 192-3). Cuando hablo de contradicciones, él las disculpa; cuando las encuentra, dice que soy demasiado generoso con ellas; cuando quiere demostrar que las juzgo mal, las contradicciones se vuelven imperdonables. El vaivén puede parecer infinito porque el párrafo siguiente comienza: "Por de pronto, las contradicciones empiezan a desvanecerse si atendemos bien a los términos que emplea Vallejo" (p. 193). Entonces, ¿de qué "abuso del lector" por parte de Vallejo nos hablaba? ¿Es o no cierto, por fin, que los libros están llenos de las afirmaciones y negaciones características de un hombre que está tratando de asumir una fe política sin traicionar sus más hondas creencias poéticas? De paso, debo señalar una errata en su cita: escribí "Vallejo se ve envuelto" no "se $h a$ envuelto", matiz que no deja de ser significante si se alude a una presunta "irresolución".

Después de señalar los tres tipos de arte que Vallejo reconoce (arte burgués, arte bolchevique o proletario, arte socialista), el Prof. McDuffie escribe: "Sin percibir tales distinciones, Oviedo llega a la calificación siguiente: 'Se diría que en El arte y la revolución trata de elaborar una teoría marxista del arte que no es sino un pálido ensayo de lo que su poesía pone admirablemente en ejercicio, es decir, la verdadera teoría de Vallejo es su praxis poética'. Pero, en el fondo, la definición vallejiana del arte socialista, o sea, del arte verdadero, es su teoría de siempre, hecho que justifica la presencia en El arte y la revolución de varios textos publicados anteriormente, textos en los que podemos estudiar la poética de Vallejo antes de su adhesión al marxismo" (p. 197). Como de costumbre, el mejor argumento que tengo para probar que el Prof. McDuffie se confunde es otra afirmación suya, páginas más adelante: "Sin duda la pasión política de Vallejo lo llevó peligrosamente cerca de una negación terminante de la libertad estética del artista, pero se salvó en último término, no sólo por medio de las salvedades aludidas, sino por el ejemplo de su propia praxis poética" (p. 201). Como de costumbre también estamos de acuerdo en algo: la praxis poética de Vallejo salva las contradicciones (o tentaciones) ideológicas en las que cayó. Pero discrepamos profundamente en cuanto a creer que el Vallejo de la época de Trilce (el que escribe en 1922 a Antenor Orrego: "Siento gravitar sobre mí una hasta ahora desconocida obligación sacratísima, de hombre y de artista, la de ser libre!') sea intelectualmente el mismo que en estos libros pone entre paréntesis la libertad creadora del artista revolucionario con propuestos como: "La forma del arte revolucionario, debe ser lo más directa, simple y descarnada posible. Un realismo implacable. Elaboración mínima... Arte de primer plano. Fobia a la media tinta y al matiz. Todo crudo, - ángulo y no curvas, pero pesado, bárbaro, brutal, como en las trincheras" (El arte y la revolución, p. 123-4). Ya se sabe que hay otros pasajes en el libro que atenúan la violencia de esta afirmación y, sobre todo, que Vallejo se negó a dar cabida en su obra poética a ese tipo de arte primario y didáctico. 
Hay otras alusiones y citas que suponen lecturas discutibles del Prof. McDuffie, pero temo ser ya demasiado engorroso si incento comentarlas todas. Me gustaría, sí, agregar que me llamó mucho la atención que un art́ículo como el suyo, tan crítico del mío, no tuviese reparos en utilizar sin comillas expresiones o formulaciones que me pertenecen. Hablando de Cocteau y de su Rappel à l'ordre, escribí: "Recojamos de ese párrafo las ideas claves-horror a mezclar la literatura con la impura realidad, gusto por las formas perfectas y gratuitas, escéptico individualismo, divinización del artista-de un escritor que, a nombre del sacerdocio de su métier, se niega a aceptar las urgencias de la historia" (p. 3). El Prof. McDuffie me glosa así: "Cocteau nos presenta al artista divinizado, egoísta, de 'buen gusto', quien busca las formas perfectas y gratuitas, sin mezclar la literatura con la impura realidad y quien, por eso, rechaza las urgencias de la época" (p. 180). En otra parte señalo que los citados libros de Vallejo "constituyen los dos primeros volúmenes de sus futuras Obras completas, que continuarán con tomos dedicados a su poesía, su narración, su teatro, etc." y que esta exhumación de inéditos "contribuye a mostrar mejor su definitiva imagen literaria y humana" (p. 4-5). Aquí, el Prof. McDuffie, es más textual y dice: "Constituyen los dos primeros volúmenes de sus futuras Obras completas, que continuarán con tomos dedicados a su poesía, su narración, su teatro, etc. y representan en sí un gran paso hacia una definitiva imagen literaria y humana de Vallejo" (p. 202). Si el autor me hubiese citado en el resto de su artículo con la misma precisión con que lo hace en esos párrafos, con mucho gusto me habría ahorrado esta respuesta.

Indiana University, Bloomington

JOSE MIGUEL OVIEDO

\section{SOBRE VALLEJO: RESPUESTA A JOSE MIGUEL OVIEDO}

El mayor error del profesor Oviedo es creer que yo opino que su artículo se caracteriza principalmente por afirmaciones y observaciones "erróneas, inexactas o confusas" (los calificativos son suyos). Siempre he tenido y tengo el mayor respeto por la labor crítica del profesor peruano, y no menos en este caso. Pero tal respeto no impide que pueda yo discrepar en algunos aspectos básicos de la valoración que él da de las dos obras de Vallejo objeto de estas discusiones. Por su parte, afirma que mi mayor error ha sido (cito textualmente) "creer que él ha visto lo que yo he visto y suponer, por lo tanto, que estamos en desacuerdo".

Lamento, en primer lugar, que la extensión de mi artículo (26 páginas) y sobre todo el "abundante despliegue de citas y notas a veces extensísimas" le moleste tanto. El artículo trata de una época importantísima en la vida intelectual de Vallejo, época además no bastante bien conocida y entendida; mientras las notas tratan de aclarar, cuando sea posible, aspectos bibliográficos 
bastante confusos (el profesor Oviedo no hace caso de mi reconocimiento de sus propios esfuerzos para aclarar la maraña bibliográfica de esta última etapa literaria de Vallejo). Creo, además, que el artículo resulta comprensible sin las notas, al parecer "demasiado aparatosas" para él.

Son dos los casos en los que, por comprender poco de su artículo (según él), he querido oponer mis "propios descubrimientos" a los del profesor Oviedo, aunque en realidad "tal desacuerdo no existe principalmente sino en la maraña de las citas". En la nota 8 de la p. 181, afirmo: "José Miguel Oviedo no parece entender bien este pasaje cuando lo califica de 'rétroceso' a los acentos de Trilce y lo contrapone a otros en que 'razona dialécticamente' Vallejo. Es nuestro juicio que este pasaje es también dialéctico, como explicamos luego". El profesor Oviedo supone, errónearnente, que no estoy de acuerdo con lo de "los acentos de Trilce". Si fuera así, icitaría seguidamente varios versos de Trilce para demostrar que, como destaco al final del artículo (p. 202), "el gran valor de lo absurdo, postulado básico desde Trilce, encuentra ya su base filosófica por excelencia en el materialismo dialéctico: todo instante de lo absurdo viene a ser lo que Vallejo llama en $R$ usia ante el segundo plan quinquenal '...una contradicción dialéctica...una contradicción en movimiento y en marcha hacia una solución' ". Esta frase, por lo demás, tiene cierta semejanza con la siguiente del mismo Vallejo: "Crucificados en vanas camisas de fuerza, avanzan así las diferencias de hojas alternas hacia el panteón de los grandes acordes", frase que yo interpreto como otra imagen de las mismas contradicciones dialécticas "en marcha hacia una solución".

Sea adecuada o no mi interpretación, la verdad es que el profesor Oviedo encuentra una vacilación intelectual de parte de Vallejo. Si no, icómo explicar el pasaje siguiente?

Vallejo...afirma [las "respuestas sin preguntas, que son el espíritu del arte y la conciencia dialéctica de las cosas"] entre avances y retrocesos que testimonian la complejidad del proceso en el que, como artista y como hombre, se ve envuelto. Si en "Individuo y sociedad" razona dialécticamente ("A tal punto es social y solidaria la conciencia individual... A tal punto el individuo es libre e independiente de la sociedad", pp. 28-29), en otras páginas todavía se escuchan acentos de Trilce: “ Ay del que logra cristalizar un gran disparate! Crucificados en vanas camisas de fuerza, avanzan así las diferencias de hojas alternas hacia el panteón de los grandes acordes" (p. 39).

¿Es o no es el propósito de este pasaje el de destacar los "avances y retrocesos" vallejianos? Bien puedo equivocarme en cuanto al aspecto dialéctico que encuentro en este texto de Vallejo, pero tal hipotético error no da razón al profesor Oviedo para escamotear el sentido básico de sus observaciones, como hace cuando dice "lo que me interesaba señalar" fue que "ese texto...recuerda Trilce". Cualquier lector mediano tendrá que reconocer su acento trílcico, sin que por eso tenga que compartir el juicio de que queda demostrada así una vacilación intelectual ideológica de parte del autor. 
En cuanto a la interpretación mía, existe además "evidencia contextual" de que Vallejo ha querido expresar una actitud dialéctica al titular la sección del libro en que figura este pasaje "Negaciones de negaciones", o sea, una alusión a lo positivo sacado de lo negativo. Dice Vallejo en un artículo que data de 1925, es decir de esos mismos años, al definir el "liberalismo verdadero": "No significa una moral defensiva o de frío eclecticismo, sino un temperamento ofensivo, pungente, que logra convertir, en la fragua interior, lo contrario y adverso, en elemento de gran amplificación de si mismo" ("Crónica de París", Artículos olvidados, p. 20). Es un sentimiento trílcico: “No subimos acaso para abajo?" (Trilce LXXVII). Es precisamente por esta continuidad de temas por lo que niego un vaivén intelectual de parte de Vallejo.

Pero el profesor Oviedo encuentra un vaivén también en mis opiniones sobre estos "avances y retrocesos" ideológicos. Por una parte, si discuto su afirmación sobre tales contradicciones ideológicas, por la otra no sólo señalo que él las juzga "con la mayor generosidad" (juicio que no implica el ser "demasiado generoso", como parafrasea luego él), sino que también encuentro argumentos para "condenar severamente esas contradicciones". Igual que el artículo del profesor Oviedo, el mío está escrito "en un lenguaje sencillo". De aquí que me resulte extraño la no comprensión en un crítico de probada sagacidad como la del profesor Oviedo.

El pasaje indicado no presenta misterio alguno; digo sencillamente que si existieran tales contradicciones, resultarían no sólo descuidos muy grandes de parte de Vallejo, sino hasta un abuso del lector, y por eso digo que las trata con suma generosidad el profesor Oviedo (juicio además que no parece precisamente peyorativo). En seguida digo: 'Pero de pronto ['por de pronto' es errata] las contradicciones empiezan a desvanecerse si atendemos bien a los términos que emplea Vallejo", y sigo con una explicación de los distingos que hace Vallejo entre algunos términos claves, precisamente para poder comprobar que, en el sistema ideológico vallejiano, no existen tales contradicciones. Pero el profesor Oviedo no se interesa por mi explicación, sino por la aparente contradicción en la que me he envuelto por contradecirle: “¿De qué 'abuso del lector' por parte de Vallejo nos hablaba?" (De ninguno, como se ve con facilidad.) Otra vez, el profesor Oviedo ha evitado o escamoteado el sentido central del pasaje al darle un sentido opuesto al verdadero.

Algo semejante ocurre cuando me cita con respeto a la llamada salvación literaria de Vallejo por medio de su praxis poética. A pesar de citarlas, el profesor Oviedo evita comentar mis palabras sobre los distingos aludidos para luego "demostrar", con evidente satisfacción, que estoy tan confundido que no me doy cuenta de que en esto estoy de acuerdo con él. Pero vale citar una vez más las palabras del profesor Oviedo: "Se diría que en El arte y la revolución trata de elaborar una teoría marxista del arte que no es sino un pálido ensayo de lo que su poesía pone admirablemente en ejercicio, es decir, la verdadera teoría de Vallejo es su praxis poética". Como siempre, no ve que Vallejo no trata de elaborar una 
teoría del arte, sino tres, o sea, una teoría del arte proletario o bolchevique, una teoría socialista (en el sentido especial que da Vallejo a esta palabra) y una teoría burguesa (no emplea nunca el término marxista respeto a sus propias teorías, más bien condena a los críticos marxistas que "observan un criterio tan arbitrario, casuístico y anarquizante, como el de cualquier esteta burgués" (El arte y la revolución, p. 34). Por medio de estas teorías, Vallejo encuentra una base teórica que pueda justificar su deseo de emplear los instrumentos de la cultura literaria para los fines de la revolución, sin negar a la vez la libertad y autonomía esencial del artista.

Por fin llega el profesor Oviedo al meollo del asunto cuando dice que discrepa "profundamente en cuanto a creer que el Vallejo de la época de Trilce...sea intelectualmente el mismo que en estos libros pone entre paréntesis la libertad creadora del artista revolucionario..." Además de lo insólito de esperar que un hombre de una vida intelectual tan intensa como Vallejo sea "intelectualmente el mismo" que el de ocho años antes, no es muy claro en qué sentido haya cambiado, según el profesor Oviedo. ¿Debo suponer que el Profesor Oviedo quiere decir que Vallejo se encuentra momentariamente despistado, poseído de una furia revolucionaria que por poco le traiciona como artista?

Pero por querer plasmar una teoría del arte más compleja que dé 'cabida a sus fines políticos tanto como artísticos, parece evidente que Vallejo no ha perdido su integridad intelectual. Su teoría no es, en fin, "un pálido ensayo de lo que su poesía pone admirablemente en ejercicio", sino que, bien entendido, incluye la teoría de su praxis poética (lo que Vallejo llama "arte socialista") y además las de las artes bolchevique y burgués. Sin comprender esto, no se puede llegar a un concepto cabal del pensamiento vallejiano de esta época.

No puedo responder a las "otras alusiones y citas que suponen lecturas discutibles" de mi parte, puesto que no las identifica el profesor Oviedo. En cuanto a los dos ejemplos de plagio aparente por mi parte, no puedo disculparme. Es evidente que en los dos casos las formulaciones del profesor Oviedo son inmejorables, y por eso quien quiera expresar tales conceptos se ve forzosamente condenado a expresarse en términos semejantes. No cabe duda de que debiera señalar yo, por medio de comillas, las fuentes de dichas formulaciones, aunque debo confesar que no me di cuenta del plagio al escribirlos (lo que no es disculpa legítima, ni mucho menos, aunque sí un elogio inconsciente).

Por fin, he tomado al pie de la letra el subtítulo "Primera lectura..." y he querido ampliar los hallazgos valiosos del profesor Oviedo. Mi error básico no fue el señalado por el crítico, el de estar de acuerdo con él sin saberlo, sino el de suponer que una "primera lectura" implicaba otras posibles y hasta más comprensivas. Sin quitarle al profesor Oviedo el mérito que merecen sus investigaciones, me permito decir que "una definitiva imagen literaria y humana de Vallejo" es, en el fondo, lo esencial del asunto, y que todos los plagios y acuerdos inconscientes no son tan importantes como una lectura adecuada del gran poeta peruano. 
Por eso, espero que él acepte estas palabras sinceras por las que expreso mi reconocimiento de su indudable capacidad crítica, pero que a la vez se dé cuenta de la complejidad del pensamiento vallejiano, y sobre todo, de su gran integridad intelectual, integridad que nunca sacrificó a ninguna causa política. 